\title{
Progresos en la terapia del cáncer avanzado de tiroides. Bases fisiopatológicas
}

\section{Arturo Orrego Monsalve}

Médico Internista Endocrinólogo, Miembro Honorario

de la Asociación Colombiana de Endocrinología,

Diabetes y Metabolismo

Fecha de recepción: 11/08/2015

Fecha de aceptación: 15/09/2015

\section{Introducción}

$\mathrm{E}$ cáncer tiroideo diferenciado (CTD) constituye el 90\% de los cánceres tiroideos y comprende el cáncer papilar, el folicular y las variedades pobremente diferenciadas histológicamente. La incidencia de los cánceres tiroideos está en continuo aumento, especialmente en mujeres ${ }^{(1)}$; la supervivencia a largo plazo es excelente y la mayoría de los pacientes mueren de otras causas. El consenso más aceptado recomienda que la mayoría de los pacientes con cáncer tiroideo clínicamente significativo deben ser sometidos primero a cirugía con tiroidectomía total y después a terapia ablativa con yodo radioactivo si el paciente está en riesgo alto de recurrencia tumoral o muerte $\mathrm{e}^{(2,3)}$. Se debe administrar terapia con levotiroxina para tratar el hipotiroidismo posquirúrgico, a dosis altas, suficientes para suprimir la TSH con el fin de eliminar el poder estimulante de la tirotropina sobre los restos microscópicos de las células tumorales, en los pacientes con alto riesgo de recurrencia. Una vez que se completa el tratamiento se debe proceder al seguimiento clínico del paciente con el fin de detectar el tejido residual "in situ" o la recurrencia tumoral, con la medición de la tiroglobulina sérica, un biomarcador, y la ultrasonografía del cuello. La recurrencia local debe tratarse con cirugía, yodo radioactivo o ambos y en raros casos con radioterapia. La remisión bioquímica completa puede presentarse en $25 \%$ a $75 \%$ de los pacientes con enfermedad recurrente en los ganglios linfáticos, en cambio la recurrencia en el lecho tiroideo tiene peor pronóstico ${ }^{(4)}$.

Las metástasis a distancia se observan en aproximadamente el 15\% de los CDT y el 50\% se presentan en el momento del diagnóstico. El 50\% se localizan en pulmones; en los huesos el $25 \%$; en pulmones y hueso, el $20 \%$ y en otros sitios, el $5 \%$. La captación del yodo radioactivo por las metástasis se puede observar en muchos de los pacientes, pero la remisión completa sólo se observa aproximadamente en el $30 \%{ }^{(5)}$, a pesar de múltiples tratamientos.
Cuando la terapia con ${ }^{131}$ I para metástasis locales o a distancia es inapropiada, se denominan como "resistentes", "refractarias", "no responden" o no "ávidas". Los tres primeros términos indican que la terapia con yodo radioactivo es de esperar que no produzca efectos significativos. El último término sugiere que las células tumorales no son capaces de captar suficiente cantidad de yodo radioactivo para dar origen a gammagrafías útiles como diagnóstico o evaluación posterapia. Sin embargo, por varias razones biológicas, los tumores pueden conservar la avidez por el ${ }^{131}$ I y no recibir suficiente dosis de radiación capaz de producir efectos clínicos significativos, como puede ocurrir cuando la depuración renal del yodo radioactivo es demasiado rápida y la retención por las células tumorales es deficiente o el cáncer es resistente a los efectos del yodo radioactivo. No debe olvidarse que los tumores que no son ávidos por el yodo radioactivo difícilmente se benefician de este material. Existen pacientes en los cuales el tumor es ávido por el yodo radioactivo y responden a la terapia, pero en quienes existen más riesgos que beneficios, un ejemplo son los pacientes con fibrosis pulmonar inducida por radiación previa o supresión medular. En la práctica pueden definirse los pacientes como resistentes a la terapia con yodo radioactivo, si presentan alguno de los siguientes criterios: 1 ) si no existe suficiente captación del ${ }^{131}$ I por el tumor o las metástasis detectadas por los métodos radiológicos usuales; 2) Tumores que aparecen o progresan radiológicamente después de una terapia reciente con yodo radioactivo, por ejemplo un año, independiente del grado de captación del ${ }^{131} I^{(6)}$. Como criterios adicionales de resistencia al yodo radioactivo, está la administración previa acumulativa de al menos $600 \mathrm{mCi}$ de ${ }^{131} \mathrm{I}^{(5)}$ y la presencia de captación alta de la fluordesoxiglucosa por el tumor en la gammagrafía con PET.

En cuanto a la enfermedad metastásica, las cirugías paliativas, la radioterapia externa o la fotocoagulación con láser pueden ofrecer mejorías parciales. Las metástasis óseas son difíciles de tratar, producen importante morbilidad y mortali$\mathrm{dad}^{(7)}$. Como en otras entidades malignas, los bifosfonatos y el denosumab pueden reducir las fracturas óseas y disminuir el dolor ${ }^{(8,9)}$. Sin embargo, no existen trabajos extensos comparativos aleatorios que demuestren con claridad la reducción de los eventos óseos con la administración de bifosfonatos o de- 
nosumab en pacientes con cáncer tiroideo avanzado. Otras terapias paliativas pueden beneficiar a algunos de los pacientes seleccionados como la vertebroplastia, la resección quirúrgica, la embolización transarterial y las técnicas de ablación con catéter ${ }^{(10,11)}$. Como en otros pacientes con avanzadas metástasis carcinomatosas, el control del dolor, el cuidado nutricional y otras medidas de soporte no curativas pueden mejorar el estilo de vida del paciente y hacer más llevadera la enfermedad antes del desenlace final.

Para los pacientes con metástasis de carcinoma diferenciado de tiroides con progresión de la enfermedad, a pesar de las terapias convencionales aceptadas, la quimioterapia de uso tradicional ha sido de poca utilidad pero sí de considerable morbilidad ${ }^{(12)}$. Informes preliminares dieron a conocer la utilidad de la monoterapia con doxorubicina, un inhibidor de la síntesis del ADN y ARN, en las metástasis del carcinoma tiroideo $^{(13,14)}$. La efectividad de este citotóxico se observó preferencialmente en las metástasis pulmonares y menos en las óseas; como efectos secundarios de esta droga, se observaron cardiomiopatía, granulocitopenia, infecciones, náuseas, vómitos, infertilidad y alopecia.

En vista de la poca utilidad y alta morbilidad de los citotóxicos utilizados en la terapia del cáncer avanzado de tiroides se vienen investigando nuevos medicamentos ${ }^{(15)}$. De importancia ha sido la identificación de mutaciones oncogénicas específicas que aparecen tempranamente en el desarrollo del cáncer tiroideo y de la comprensión de la interacción de las células tumorales y el microambiente celular, especialmente la red vascular ${ }^{(6)}$ (figura 1 y 2).

\section{Características genéticas, moleculares y biológicas de los cánceres tiroideos diferenciados}

En los últimos 20 años el conocimiento de las bases genéticas y biológicas de los cánceres derivados de la célula folicular ha cambiado grandemente con la proposición de un modelo molecular en la progresión del cáncer tiroideo(16).

\section{Tumorogénesis del cáncer tiroideo diferenciado}

La vía oncogénica principal relacionada con la iniciación y progresión de los cánceres tiroideos es la RAS/RAF/MAPK (proteína cinasa mitogénica activada) y la PI3K/Akt (la fosfatidilinositol 3-cinasa), gracias a la importancia en la formación, diferenciación y supervivencia de las células tumorales ${ }^{(16)}$ (figuras 1 y 2).

Estas mutaciones, sin embargo, no pueden ser la causa única del comportamiento agresivo del carcinoma papilar pobremente diferenciado porque la proporción de estos cánceres con mutaciones BRAF es más alta que en aquellos cánceres con características biológicas de alto riesgo (invasión extensa, recurrencia, metástasis a distancia y mortalidad debida a es- tos tumores). El cáncer folicular tiene una etiología molecular diferente (figuras 1 y 2 ).

Finalmente, el carcinoma tiroideo diferenciado está asociado con menos síndromes genéticos por pérdida de la función supresora del tumor (síndrome de Cowden, síndrome PTEN, complejo de Carney, etc.).

Histopatológicamente, el cáncer tiroideo poco diferenciado está caracterizado por un patrón infiltrativo de crecimiento, necrosis, alto índice mitótico e invasión vascular. El carcinoma tiroideo diferenciado avanzado se define más por su comportamiento que por su histología. Estos tumores son generalmente resistentes al yodo radioactivo, positivos con el FDG-PET (tomografía con emisión de positrones, utilizando fluordesoxiglucosa), invasivos localmente y pueden dar origen a metástasis a distancia. Aunque los cánceres tiroideos anaplásicos (CTA) y los pobremente diferenciados (CTPD) producen mayor mortalidad que los carcinomas tiroideos diferenciados,

Figura 1. Perfil de mutaciones en el cáncer tiroideo

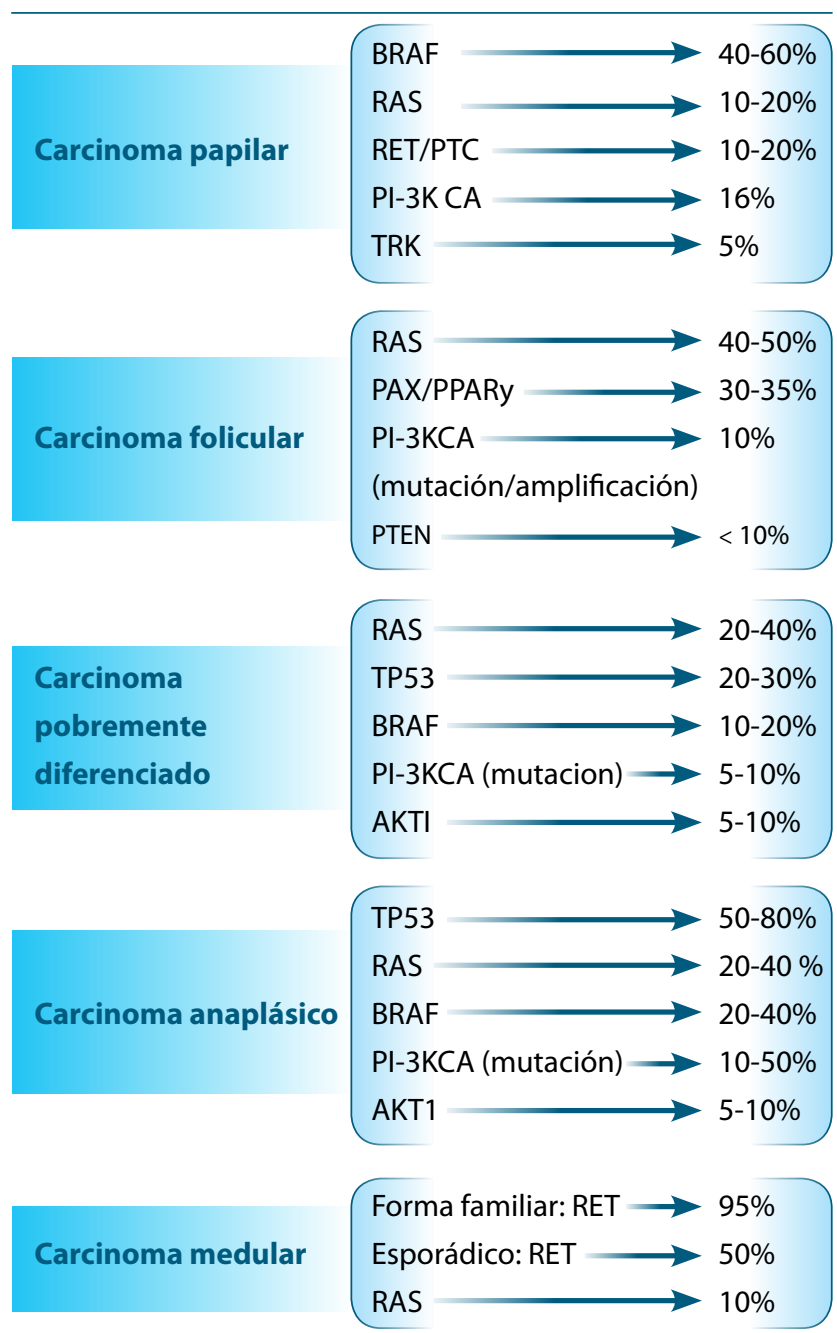

BRAF, fibrosarcoma tipo rápidamente activado;RAS, sarcoma de rata; RET, reorganizado durante la transfección; PI-3KCA, amplificación de la cinasa 3 fosfatidilinositol; PAX8/PPARy, receptor gama activado perixomal; TRK, receptor de a tirosina cinasa;PTEN, homólogo de las fosfatasa y tensina; AKT1, proonéogeno viral. 
Figura 2. A: Defectos moleculares de las neoplasias tiroideas, desarrollo y progresión (modificada de referencia

6) B: cáncer tiroideo avanzado, invasivo, metastásico, resistente al 131I, PET positivo
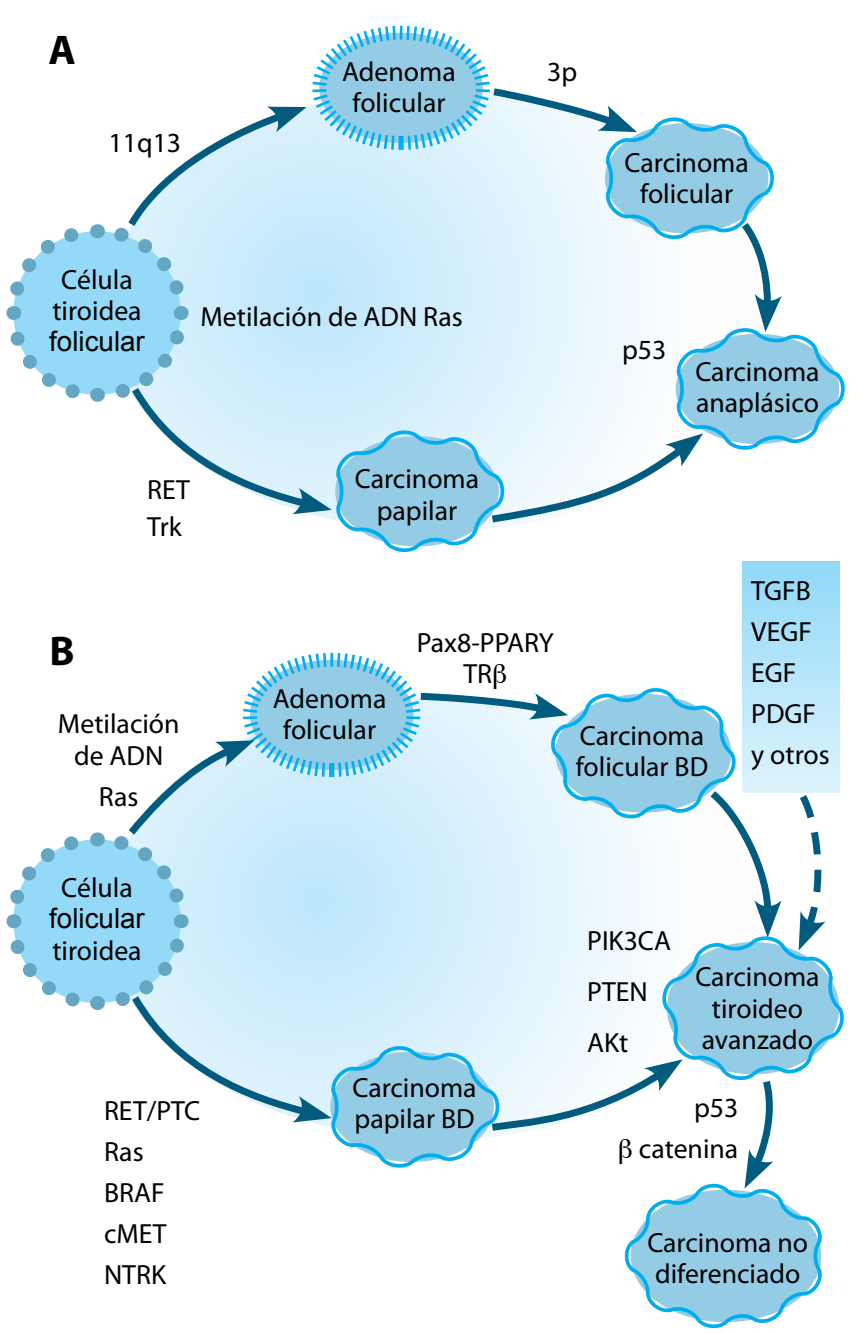

RET, reorganizado durante transfección; TrK, tropomicina, receptor de la cinasa; 11q13, brazo largo del cromosoma 11; 3p, brazo corto del cromosoma 3; TRB receptor beta tiroideo; MET, protooncógeno; RET/PTC, carcinoma papilar tiroideo;NTRK, Trk, familia de genes; PI-K3CA, fosfatidilinositol cinasa 3; PTEN, fosfotensin; CTBD carcinoma tiroideo bien diferenciado; TGF $\beta$, factor de crecimiento tumoral beta; VEGF, factor de crecimiento endotelial vascular; EGF, factor de crecimiento epidermoide; PDGF, factor de crecimiento del papilar diferenciado; RAS, sarcoma de rata; carcinoma folicular y papilar BD, bien diferenciado.

la mayoría de los 1780 casos de cáncer tiroideo que se calculó que debían morir en el año 2013, eran cánceres tiroideos diferenciados. Rivera y colaboradores ${ }^{(17)}$ examinaron las metástasis de pacientes resistentes al yodo, PET positivos e invasivos y encontraron que el $80 \%$ de estos tumores presentaban una histología agresiva. De forma interesante se observó que con frecuencia las metástasis distantes de carcinoma diferenciado de tiroides habían progresado a un carcinoma tiroideo menos diferenciado histopatológicamente en el $70 \%$ de los $\operatorname{casos}^{(17)}$. La activación de la vía MAPK (proteína cinasa mitogénica activada) es común en los carcinomas tiroideos bien diferencia- dos, en los pobremente diferenciados, en los casos avanzados y en los anaplásicos ${ }^{(6,17,18)}$. Aunque este hallazgo puede sugerir las posibilidades de ser utilizado como blanco terapéutico, no existe evidencia de que este hecho pueda explicar las diferencias existentes entre el carcinoma tiroideo bien diferenciado, el avanzado o el pobremente diferenciado ${ }^{(6,18)}$.

Genéticamente, con frecuencia en los cánceres tiroideos avanzados se encuentra mutación de la secuencia PI-3KCA (cinasa-3 activada fosfatidilinositol), más en los carcinomas tiroideos anaplásicos que en los bien diferenciados ${ }^{(19)}$. Además el $35 \%$ de los tumores con mutaciones PI-3KCA presentaban BRAF y $30 \%$ de estos tumores tenían mutaciones de PI-3KCA y RAS. Ricarte-Filho y colaboradores ${ }^{(20)}$ identificaron 111 mutaciones en pacientes con cáncer avanzado de tiroides, resistentes a ${ }^{131} \mathrm{I}$.

Un hecho único en los cánceres avanzados de tiroides es la inactivación del supresor p53, mucho más frecuente en los pobremente diferenciados y en los anaplásicos.

Como interesante, con métodos especiales de biología molecular se demostró que los carcinomas anaplásicos expresaban más VEGF (factor de crecimiento vascular) y B-cateninas, lo que sugería que estos factores podían ser importantes en la formación y mantenimiento de los cánceres tiroideos avanzados y que podrían servir de blanco en la terapia tumoral(21). Este grupo demostró que la mutación p53 era más frecuente en los carcinomas tiroideos anaplásicos y en los pobremente diferenciados ${ }^{(22,23)}$.

La teoría que lidera los mecanismos que explican las diferencias entre el cáncer tiroideo bien diferenciado (CTBD), el diferenciado (CTD), el pobremente diferenciado (CTPD) y el anaplásico (CTA) se denomina "múltiple hit". El CDT se inicia por mutación o cambios en la MAPK (proteína cinasa mitogénica activada) o la vía PAX-8-PPAR y mutaciones adicionales (PI-3k/VEGFR fosfatidilinositol-cinasa3/receptor del factor de crecimiento endotelial vascular) y otros factores que llevan a una enfermedad más agresiva ${ }^{(24)}$ (figura 1).

Se ha propuesto otra teoría para explicar la diferencia de agresividad en los cánceres tiroideos: el modelo de la carcinogénesis fetal. En este modelo los CTBD se derivan de los protirocitos y tiroblastos, en cambio los CTA se originan en los precursores de las stem cell. La persistencia de estas células y la formación de carcinomas tiroideos a partir de ellas dan origen a recurrencias más frecuentes del tumor tiroideo y a un peor pronóstico ${ }^{(25)}$. Visko y colaboradores ${ }^{(26)}$ encontraron, comparando el frente invasivo con el centro del tumor, que la porción externa tumoral habría transformado las células epiteliales en mesenquimales; según estos autores es necesaria esta transformación para que el tumor invada y dé origen a metástasis. En una estudio comparativo de 12 pacientes que presentaban, en el mismo tumor, cáncer diferenciado de tiroides y anaplásico, se encontró que en estas últimas células tumorales se identificaron más en el receptor del factor de crecimiento endotelial vascular que en el tumor diferenciado, lo 
que sugería que este factor era importante en la formación y mantenimiento del cáncer avanzado de tiroides y que la inhibición en su síntesis podría ser de importancia terapéutica ${ }^{(21)}$. Este grupo halló también que los niveles de la p53 eran más altos en los carcinomas tiroideos anaplásicos , tal como se había demostrado en los carcinomas tiroideos avanzados.

Knauf y colaboradores ${ }^{(27)}$ demostraron que la mutación BRAF convertía las células tiroideas en susceptibles a la acción del factor de crecimiento tumoral beta (TGFB) y que ambos factores eran necesarios para la transición a un cáncer tiroideo más avanzado. Con base en estos estudios, se comienza a visualizar que la transición de los carcinomas tiroideos diferenciados a cánceres tiroideos avanzados se debe a la combinación de factores genéticos (mutaciones, amplificación de genes y reorganización) y a factores autocrinos o paracrinos (microambiente, células estromales, células inmunes y vasculares). Aunque los cánceres tiroideos avanzados, sin lugar a dudas, son heterogéneos, la comprensión simultánea de los factores genéticos y ambientales podría ayudar a seleccionar terapias tentativas de curación o al menos de control de estos tumores agresivos.

\section{Aplicación de los conocimientos genéticos a la terapia del cáncer tiroideo avanzado}

\section{Inhibidores de las múltiples cinasas}

Dos importantes avances en la última década han hecho posible augurar mejores posibilidades terapéuticas para el cáncer avanzado tiroideo, 1) mejor comprensión de la genética y la biología molecular de estos carcinomas y 2) la factibilidad de realizar varios ensayos clínicos, multicéntricos, cooperativos, en pacientes con cáncer tiroideo avanzado. Antes se había demostrado que ciertas anormalidades en la acción de las cinasas, a través de modificaciones genéticas o disfunciones autocrinas o paracrinas, jugaban un papel primordial en el desarrollo del cáncer tiroideo avanzado. De ahí surgió la idea de que inhibidores de las cinasas podrían constituir un excelente candidato en la terapia de estos tumores tiroideos.

En la figura 3 se enumeran los inhibidores de las cinasas que se han ensayado en la terapia del cáncer avanzado de tiroides, refractarios a tratamientos convencionales y sus probables mecanismos de acción. No nos detendremos a detallar cada una de las drogas utilizadas; con este fin pueden revisar las referencias bibliográficas ${ }^{(6,18-28,29)}$.

\section{Resumen y recomendaciones terapéuticas}

A pesar de la creciente utilización de los inhibidores de las cinasas mencionados en la figura 3, el uso de estos fármacos debe ser limitado a pacientes altamente seleccionados. Los efectos secundarios por su utilización son frecuentes y muchos de ellos graves y en ocasiones fatales ${ }^{(6,18,28)}$. Como manifestaciones secundarias están: lesiones cutáneas, diarrea, náuseas, fatiga, anorexia, hipertensión, mucositis y citopenias $^{(29)}$. De
Figura 3. Inhibidores de la acción de las cinasas en las células tumorales y vasculares
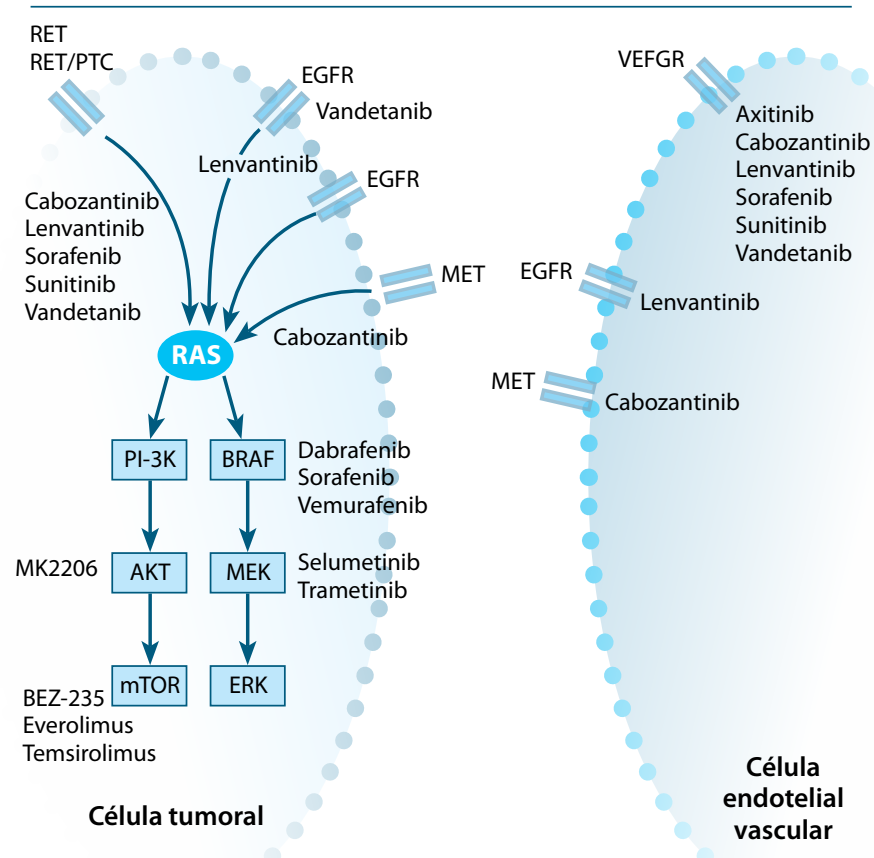

RET/PCT, carcinoma papilar tiroideo; RAS, sarcoma de rata; BRAF, fibrosarcoma tipo b-rápidamente acelerado; PI-3KCA, oncógenofosfatidilinositol 3 cinasa; $\mathrm{MEK}$, cinasa mitogénica extracelular; ERK, receptor externo de cinasa; AKT, cinasa tumoral activada; mTOR, receptor de la nopamicina; VEFGFR, receptor del factor de crecimiento endotelial vascular; EGFR, receptor del factor de crecimiento epidermoide; MET prooncógeno.

mayor importancia, pero raras están: falla cardiaca, arritmias, fístulas entéricas y perforación, hemorragias, daño hepático, renal, disfunción neurológica y cánceres escamosos. En la mayoría de los pacientes tratados se observa respuesta parcial, y puede estabilizarse la enfermedad. Sin embargo, una respuesta completa es excepcional y esta terapia no ha demostrado, hasta ahora, aumento de sobrevida de los pacientes tratados. Por todo lo anterior, esta quimioterapia debe limitarse a pacientes refractarios al yodo radioactivo, con metástasis o con lesiones locales no resecables que progresan pese a las dosis supresivas de las hormonas tiroideas. Una vez que se tome la decisión de tratar un paciente con los inhibidores de la cinasa, lo ideal sería que el paciente entrara dentro de un programa de investigación con otros pacientes, en centros con experiencia en estas drogas. Se debe conseguir autorización escrita del paciente antes de iniciar la terapia y dar a conocer los riesgos y beneficios que pueden obtenerse con este tratamiento.

Años atrás se habían informado que la monoterapia con doxorubicina, un inhibidor de la síntesis de ADN y ARN, podría ser una terapia aceptada para el cáncer tiroideo metastásico ${ }^{(13,14)}$, especialmente de mayor utilidad en metástasis pulmonares más que en las óseas, pero su toxicidad similar, en muchas manifestaciones a las observadas con los inhibidores de las cinasas, limitó su utilidad. 
Más recientemente, la FDA aprobó los inhibidores multicinasa, vandetanib y cabozantinib en el carcinoma medular metastásico, los cuales habían demostrado capacidad para detener la enfermedad progresiva y mejorar la supervivencia y que habían pasado con éxito la etapa III de la investigación.

\section{Referencias}

1. Jemal A. Bray F, Center MM, et al. Global cancer statistics. CA Cancer J Clin. 2011;61:69-90.

2. Cooper DS, Doherty GM, Haugen BR, et al. Revised American Thyroid Association Management Guidilines for Patients with Tiroid Nodules and Differentiated Thyroid Cancer. Thyroid. 2009;19:1167-1214.

3. Pacini F, Castagna MG, Brilli L, et al. Thyroid cancer: ESMO Clinical Practice Guidelines for diagnosis, treatment and follow-up. Ann Oncol. 2010;21(suppl 5) v 214- v 219.

4. Steward DL. Update in utility of secondary node dissectiom for papillary thyroid cancer. J Clin Endocrinol Metab. 2012;97:3393-3398.

5. Durante C, Haddy IV, Baudin E, et al. Long- term outcome of 444 patients with distant metastases from papillary and folicular carcinoma: benefits and limits of radioiodine therapy. J Clin Endocrinol Metab. 2006;91:28922899.

6. Haugen RB, Sherman I S. Evolving approaches to patients with advanced differentiated thyroid cancer. Endocrine Reviews. 2013; 34: 439-455.

7. Muresan MM, Oliver D , Leclere J, et al. Bone metastasis from differentiated carcinoma. Endocr Relat Cancer. 2008; 15: 37-49.

8. Vitale G, Fonderico F, Martignetti A, et al. Pamidronate improves the quiality of life and induces clinical remission of bone metastases in patients with thyroid cancer. Br J Cancer. 2001; 84: 1586-1590.

9. Orita Y, Sugitani I, Toda K, et al. Zoledromic acid in the threatment of bone metastases from differentiated thyroid carcinoma. Thyroid. 2011;21:3135 .

10. Bernier MO, Leenhard L, Hoang C, et al. Survival and therapeutic modalities in patients with bone metastases of differentiated thyroid carcinoma. J Clin Endocrinol Metab. 2001;86:1568-1573.

11. Zetting G, Fueger BJ, Passler C, et al. Long-term follow-up of patients with bone metastases from differentiated thyroid carcinoma-surgey or conventional therapy?. CLin Endocrinol (Oxf). 2002;56: 377-382.

12. Sherman SI. Citotoxic chemotherapy for differentiated thyroid carcinoma. Clin Oncol. 2010;22:4

13. Gottlieb JA, Hill CS Jr. Chemotherapy of thyroid cancer with adriamycin. Experience with 30 patients. N Engl J Med. 1974;290:193-197.

14. MatuszczyK, Petersenn S, Bockisch A, et al. Chemoterapy with doxorubicin in progressive medullary and thyroid carcinoma of folicular epithelium Horm Metab Res. 2008:40: 20-213.

15. Sherman SI. Targeted therapies for thyroid tumor. Mod Pathology. 2011;24:s44-s52.
16. Fagin JA. Genetic basic of endocrine disease 3: molecular defects in thyroid gland neoplasic. J Clin Metab. 1992;75: 1398-1400.

17. Rivera M, Ghossein RA, Schoder H, et al. Histopathological characterization of radioactive iodine-refractory fluorodeoxyglucose-positron emisión tomography-positive thyroid carcinoma. Cancer. 2008;113:48-56.

18. Gordoa TA, Diez JJ, Duran M, et al. Advances in thyroid cancer treatment: latest evidence and clinical potence. Ther Adv Med Oncol. 2015;711:22-38

19. Garcia-Rostan G. Mutation of the PIK3CA gene in anaplasic thyroid cancer Cancer Res. 2012;65: 10199- 10207.

20. Ricarte- Filho JC, Ryder M, Chitale Da, et al. Mutational profile of advanced primary and metastasic radioactive Iodine-refractary thyroid cancers reveals distinct pathogenetic roles for BRAF, PIK3CA, and AKT1. Cancer RES. 2009;69:4885-4893.

21. Wiseman SM, Griffith OL, Deen S, et al. Identification of molecular markers altered during transformation of differentiated into anaplasic thyroid carcinoma. Arch Surg. 2007;142:717-729.

22. Ito T, Seyama T, Mizuno T, et al. Unique association of $\mathrm{p} 53$ with undifferentiated but not with differentiated carcinomas of the thyroid gland. Cancer Res. 1992;52:1369-1371.

23. Fagin JA, MAtsuo K, Kamarkar A, et al.High prevalence of mutations of the p53 gene in poorly differentiated human thyroid carcinoma. J Clin Invest. 1993; 91:179-184.

24. Fagin JA, Mitsiades N. Molecular pathology of thyroid cancer: Diag nostic and clinical implications. Best Pract Res Clin Endocrinol Metab. 2008;22:955-969.

25. Takano F. Fetal cell carcino-genesis of the thyroid: theory and practice. Semin Cancer Biol. 2007; 17:233-240.

26. Vasko V, Espinosa AV, Scouter W, et al. Gene Expression and functional evidence of epitelial-to- mesenchynal transition in papillary thyroid carcinoma invasion. Proc Natl Acad Sci USA: 2007;104: 2803-2808.

27. Knauf JA, Sartor MA, Medvedovic M, et al. Progression of BRAF-induced Thyroid cancer associated with epitelial-mesenchynal transition requering concomitant MAP kinase and TGF $\beta$ signaling.Oncogene.2011;30:3153-3162.

28. Azar AF, Lee LS, Rosen EJ. Medullary thyroid cancer: an update for surgeons. The Am Surg. 2015;81:1-9.

29. Cabanillas ME, Waguespack S, Bronstein $Y$, et al. Treatment with thyrosine kinase inhibitors for patients with differentiated thyroid cancer. The MD Anderson Experience. J Clin Epidemiol Metab . 2010;95:2588-2595 